\title{
A nonabelian trace formula
}

\author{
Jayce Robert Getz ${ }^{1 *}$ and Paul Edward Herman ${ }^{2}$
}

\author{
*Correspondence: \\ jgetz@math.duke.edu \\ 1 Department of Mathematics, Duke \\ University, Durham, NC 27708-0320, \\ USA \\ Full list of author information is \\ available at the end of the article
}

\begin{abstract}
Let $E / F$ be an everywhere unramified extension of number fields with Gal $(E / F)$ simple and nonabelian. In a recent paper, the first named author suggested an approach to nonsolvable base change and descent of automorphic representations of GL2 along such an extension. Motivated by this, we prove a trace formula whose spectral side is a weighted sum over cuspidal automorphic representations of $G_{2}\left(\mathbb{A}_{E}\right)$ that are isomorphic to their Gal $(E / F)$-conjugates. The basic method, which is of interest in itself, is to use functions in a space isolated by Finis, Lapid, and Müller to build more variables into the trace formula.
\end{abstract}

2010 Mathematics subject classification: Primary 11F70, Secondary 11F66

\section{Introduction}

Let $F$ be a number field and let $H, G$ be connected reductive $F$-groups. The Langlands functoriality conjecture predicts that given an $L$-map

$$
{ }^{L} H \longrightarrow{ }^{L} G
$$

one should have a corresponding transfer of packets of automorphic representations of $H\left(\mathbb{A}_{F}\right)$ to packets of automorphic representations of $G\left(\mathbb{A}_{F}\right)$ compatible with local transfers at every place $v$ of $F$. If the rank of $G$ is large, in most cases where this conjecture is known, the image of the transfer map induced by (1.0.1) has a very simple description, namely it consists of $L$-packets fixed by a finite-order automorphism of $G$. One has a standard technique available to isolate representations that are isomorphic to their twists under an automorphism of $G$, namely the twisted trace formula, and one can compare this to a trace formula on $H$ to establish the desired transfer; see [2] for the first set of examples in arbitrary rank and [1,22] for more general examples that represent the state of the art. The alternate approach via converse theory is different, but the set of $L$-maps treated in arbitrary rank is roughly the same [5].

If one could isolate the automorphic representations of a group $G$ invariant under a noncyclic group of automorphisms, then one could hope to establish new cases of Langlands functoriality. In this paper, we take the first step in this direction.

\subsection{The trace formula}

It is convenient to first explicitly describe the trace formula we will employ. Let $F$ be a number field, let $K_{\infty} \leq \mathrm{GL}_{2}\left(F_{\infty}\right)$ be the standard maximal compact subgroup and let

$$
\mathrm{GL}_{2}\left(\mathbb{A}_{F}\right)^{1}:=\operatorname{ker}\left(|\cdot| \circ \operatorname{det}: \mathrm{GL}_{2}\left(\mathbb{A}_{F}\right) \longrightarrow \mathbb{R}_{>0}\right)
$$

(c) 2015 Getz and Herman. This is an Open Access article distributed under the terms of the Creative Commons Attribution License (http://creativecommons.org/licenses/by/4.0), which permits unrestricted use, distribution, and reproduction in any medium, provided the original work is properly cited. 
where $|\cdot|$ is the usual idelic norm. Moreover, let $B=T U \leq \mathrm{GL}_{2}$ be the standard Borel subgroup of upper triangular matrices with $T \leq \mathrm{GL}_{2}$ the subgroup of diagonal matrices, $U$ the unipotent radical of $B$ and

$$
T\left(\mathbb{A}_{F}\right)^{1}=\left\{\left(\begin{array}{cc}
t_{1} & \\
& t_{2}
\end{array}\right) \in T\left(\mathbb{A}_{F}\right):\left|t_{1}\right|=\left|t_{2}\right|=1\right\} .
$$

Using these subgroups, one defines spaces of test functions

$$
\mathcal{C}\left(\mathrm{GL}_{2}\left(\mathbb{A}_{F}\right)^{1}\right)
$$

as in $\S 4$ below. The key property of these spaces of test functions is that, although their elements are not necessarily compactly supported functions at any place, the trace formula is still valid for them, as proven in [7] (and in greater generality in [9] and [8]). Explicitly, in [7], one finds a proof of the following theorem:

Theorem 1.1 (Arthur-Finis-Lapid-Müller-Selberg). For any $f \in \mathcal{C}\left(\mathrm{GL}_{2}\left(\mathbb{A}_{F}\right)^{1}\right)$, the sum

$$
\begin{aligned}
& \sum_{\gamma / \sim} \operatorname{meas}\left(\mathrm{GL}_{2 \gamma}(F) \backslash \mathrm{GL}_{2 \gamma}\left(\mathbb{A}_{F}\right)^{1}\right) \int_{\mathrm{GL}_{2 \gamma}\left(\mathbb{A}_{F}\right) \backslash \mathrm{GL}_{2}\left(\mathbb{A}_{F}\right)} f\left(x^{-1} \gamma x\right) d x \\
& +\operatorname{meas}\left(T(F) \backslash T\left(\mathbb{A}_{F}\right)^{1}\right) \sum_{t \in T(F)} \int_{K_{\infty} \times \mathrm{GL}_{2}\left(\widehat{\mathcal{O}}_{E}\right)} \int_{U\left(\mathbb{A}_{F}\right)} f\left(k^{-1} t u k\right) \omega(t, u) d u d k \\
& +\operatorname{meas}\left(T(F) \backslash T\left(\mathbb{A}_{F}\right)^{1}\right) \sum_{t \in T(F)} \lambda_{t, S} \int_{K_{\infty} \times \mathrm{GL}_{2}\left(\widehat{\mathcal{O}}_{E}\right)} \int_{U\left(\mathbb{A}_{F}\right)} f\left(k^{-1} t u k\right) d u d k \\
& -\operatorname{tr} R_{\text {res }}(f)+\frac{1}{4 \pi} \int_{i \mathbb{R}} \operatorname{tr} M^{-1}(s) M^{\prime}(s) I(f, s) d s-\frac{1}{4} \operatorname{tr} M(0) I(f, 0)
\end{aligned}
$$

is absolutely convergent and equal to $\operatorname{tr} R_{\text {cusp }}(f)$.

Here, the sum on $\gamma$ is over elliptic conjugacy classes in $\mathrm{GL}_{2}(F)$, and $R_{\text {res }}(f)$ (resp. $\left.R_{\text {cusp }}(f)\right)$ denotes the restriction of the usual convolution operator $R(f)$ to the residual (resp. cuspidal) spectrum. Moreover, $\mathrm{GL}_{2 \gamma}$ denotes the centralizer of $\gamma$ in $\mathrm{GL}_{2}$.

If $f \in C_{c}^{\infty}\left(\mathrm{GL}_{2}\left(\mathbb{A}_{F}\right)^{1}\right)$, then this formula is a special case of the Arthur-Selberg trace formula. In general, this is ([7], Theorem 1), with a caveat: the theorem in loc. cit. is stated only for $F=\mathbb{Q}$, but this is only for convenience (see the introduction of [7]). We will not explicate the definition of the undefined terms and measures in the expression above; instead, we refer the reader to loc. cit. From a qualitative point of view, the key point is that the first line of the equality in Theorem 1.1 is equal to $\operatorname{tr} R_{\text {cusp }}(f)$ up to "error terms", i.e., the rest of the expression.

\section{Remarks.}

(1) The result is actually better than stated above, for the terms in the trace formula define continuous seminorms on $\mathcal{C}\left(\mathrm{GL}_{2}\left(\mathbb{A}_{F}\right)^{1}\right)$ (see [7], Theorem 1). In particular, it is shown in loc. cit. that the trace norm of $\operatorname{tr} R_{\text {cusp }}(f)$ is bounded.

(2) The reader may protest that we have not defined most of the distributions occurring in Theorem 1.1. This choice was made for two reasons. First, it would unnecessarily add to the length of this paper. Second, we are actually only using Theorem 1.1 as motivation. The content of our main theorem, Theorem 1.2 below, is the construction of a particular function in $\mathcal{C}\left(\mathrm{GL}_{2}\left(\mathbb{A}_{F}\right)^{1}\right)$ and a computation of how it acts on the cuspidal spectrum. The point of stating the trace formula of Theorem 1.1 
is to emphasize that one can substitute our test function into the trace formula and obtain a meaningful, absolutely convergent geometric expression for its trace using Finis, Lapid, and Müller's extension of the Arthur-Selberg trace formula.

\subsection{The main theorem}

Let $E / F$ be an everywhere unramified Galois extension split at all infinite places with

$$
\operatorname{Gal}(E / F)=\langle\iota, \tau\rangle
$$

where $|\iota|=2$ and $\tau$ has odd order.

Remark. Any nonabelian finite simple group can be generated by an involution and an element of odd order by a result of Guralnick and Malle (see [13], Theorem 1.6 and the paragraph after it).

Let

$$
F^{\prime}:={ }^{\iota=1} E \quad \text { and } \quad F_{0}:={ }^{\tau=1} E
$$

denote the fixed fields of $\iota$ and $\tau$, respectively. We let

$$
\eta: F^{\prime \times} \backslash \mathbb{A}_{F^{\prime}}^{\times} \longrightarrow \mathbb{C}^{\times}
$$

denote the Kronecker character attached to the extension $E / F^{\prime}$. We also let

$$
G:=\operatorname{Res}_{\mathcal{O}_{E} / \mathcal{O}_{F}} \mathrm{GL}_{2} \text { and } G_{0}:=\operatorname{Res}_{\mathcal{O}_{F_{0}} / \mathcal{O}_{F}} \mathrm{GL}_{2} .
$$

Let $K_{\infty} \leq G\left(F_{\infty}\right)$ be the standard maximal compact subgroup and let

$$
K^{\infty}:=G\left(\widehat{\mathcal{O}}_{F}\right) \leq G\left(\mathbb{A}_{F}^{\infty}\right) .
$$

As usual, we set $K=K_{\infty} K^{\infty}$. Finally, let $S$ be a $\operatorname{Gal}(E / F)$-invariant set of places of $E$ containing all infinite places, all places where $E / \mathbb{Q}$ is ramified, and all dyadic places. We also denote by $S$ the set of places of any subfield $E \geq k \geq F$ dividing $S$.

Let $\Psi \in C_{c}^{\infty}\left(G\left(\mathbb{A}_{F}\right)^{1} / / K\right)$. In $\S 5$ we define certain functions

$$
V:=V_{\Psi} \in \mathcal{C}\left(G\left(\mathbb{A}_{F}\right)^{1}\right) \quad \text { and } \quad b_{E / F_{0}} V \in \mathcal{C}\left(G_{0}\left(\mathbb{A}_{F}\right)^{1}\right)
$$

such that the following theorem holds:

Theorem 1.2. Let $f \in C_{c}^{\infty}\left(G\left(\mathbb{A}_{F}\right)^{1}\right)$. Consider

$$
2 \sqrt{d}_{E} \sum_{\Pi \cong \Pi_{\Pi \tau} \Pi} \operatorname{tr} \Pi(f * \Psi) \alpha^{\Pi_{S}^{\prime}}\left(W_{0 S}\right) L^{S}\left(1, \Pi^{\prime} \times \Pi^{\prime \vee} \times \eta\right),
$$

where the sum is over cuspidal automorphic representations of $G\left(\mathbb{A}_{F}\right)^{1}$ spherical at every place, $\Pi^{\prime}$ is a cuspidal automorphic representation of $\mathrm{GL}_{2}\left(\mathbb{A}_{F^{\prime}}\right)^{1}$ whose base change to $E$ is $\Pi$, and the L-function is the partial Rankin-Selberg L-function.

The sum (1.2.2) is absolutely convergent. If $f^{G_{0}} \in C_{c}^{\infty}\left(G_{0}\left(\mathbb{A}_{F}\right)^{1}\right)$ is a transfer off then (1.2.2) is equal to

$$
\frac{1}{\left[E: F_{0}\right]} \operatorname{tr} R_{\text {cusp }}\left(f^{G_{0}} * b_{E / F_{0}}(V)\right) .
$$


Here, $W_{0 S} \in \mathcal{W}\left(\pi_{S}, \psi_{S}\right)$ is a Whittaker newform (see $\left.\S 3.1\right)$ for $\Pi$ and $\alpha^{\Pi_{S}^{\prime}}\left(W_{0 S}\right)$ is defined as in (5.1.8). Our conventions regarding transfers of functions are described in $\S 4$.

The proof of Theorem 1.2 boils down to carefully constructing the function $V$ and then proving that it lies in $\mathcal{C}\left(G\left(\mathbb{A}_{F}\right)^{1}\right)$. We will explain this in broad terms in $\S 1.5$ below.

\subsection{Building more variables into the trace formula}

Even without the application to nonabelian trace formulae, the method used to prove Theorem 1.2 is of interest in itself. We explain it briefly here. Let $\Psi \in C_{c}^{\infty}\left(G\left(\mathbb{A}_{F}\right)^{1} / / K\right)$ and let $g \in G\left(\mathbb{A}_{F}\right)$. Consider the function

$$
\Psi_{g}:=\sum_{y \in E^{\times}} \Psi * \mathbb{W}\left(\left(\begin{array}{ll}
y \\
1
\end{array}\right) g\right)
$$

with $\mathbb{W}_{g}$ defined as in $\S 3$. A slight variant of the proof of Proposition 5.2 implies that $\Psi_{g} \in$ $\mathcal{C}\left(G\left(\mathbb{A}_{F}\right)^{1}\right)$, and corollaries 3.2 and 3.5 imply that for everywhere spherical automorphic representations $\pi$ with Whittaker newform $\varphi_{0}$ one has

$$
K_{\pi\left(\Psi_{g}\right)}\left(g_{1}, g_{2}\right):=\sum_{\varphi \in \mathcal{B}(\pi)} \pi\left(\Psi_{g}\right) \varphi\left(g_{1}\right) \bar{\varphi}\left(g_{2}\right)=\frac{\sqrt{d}_{E} \pi(\Psi) \varphi_{0}(g) \varphi_{0}\left(g_{1}\right) \bar{\varphi}_{0}\left(g_{2}\right)}{\left\|\varphi_{0}\right\|^{2}}
$$

where $\mathcal{B}(\pi)$ is an orthonormal basis of the $\pi$-isotypic subspace of the cuspidal subspace of $L^{2}\left(G(F) \backslash G\left(\mathbb{A}_{F}\right)^{1}\right)$. This fact can be used to build trace formulae with interesting weight factors. One possibility that comes to mind is applying a variant of the construction of $V$ and integrating over the diagonal maximal torus to build a trace formula whose spectral expansion is weighted by central values of the standard $L$-function.

We also feel that the construction of $\mathbb{W}_{g}$ at the archimedian places in $\S 3.3$ is of interest in its own right, for it amounts to an archimedian analogue of the Casselman-Shalika formula in a very special case. It would be interesting to generalize it general $K_{\infty}$-types and to $\mathrm{GL}_{n}$ over archimedian fields.

\subsection{Possible applications}

The authors view Theorem 1.2 as a nontrivial step towards proving the existence of base change and descent of automorphic representations of $\mathrm{GL}_{2}$ along the extension $E / F$. We illustrate this with a typical example. Assume that $\operatorname{Gal}(E / F)$ and $\operatorname{Gal}(E / F)=\langle\iota, \tau\rangle$ with $|\iota|=2$ and $|\tau|$ odd. One does not expect all cuspidal representations $\pi$ of $\mathrm{GL}_{2}\left(\mathbb{A}_{E}\right)$ that are $\mathrm{Gal}(E / F)$-invariant to descend to $\mathrm{GL}_{2}\left(\mathbb{A}_{F}\right)$, but Langlands functoriality predicts that one can choose a finite set of characters $\chi: E^{\times} \backslash \mathbb{A}_{E}^{\times} \longrightarrow \mathbb{C}^{\times}$depending on $\operatorname{Gal}(E / F)$ such that if $\pi$ is $\operatorname{Gal}(E / F)$-invariant then $\pi \otimes \chi$ descends to $\mathrm{GL}_{2}\left(\mathbb{A}_{F}\right)$ for some $\chi$ ([11], Proof of Lemma 5.1). This additional complication disappears if we assume in addition that $\operatorname{Gal}(E / F)$ is the universal perfect central extension of a finite simple group (compare loc. cit.). Thus, let us assume that $\operatorname{Gal}(E / F)$ is a universal perfect central extension of a finite simple group. For simplicity, we exclude the case where $\mathrm{Gal}(E / F)$ is the universal perfect central extension of the icosahedral group (this case is treated from a slightly different perspective in [11]).

Let

$$
f \in C_{c}^{\infty}\left(G\left(\mathbb{A}_{F}\right)^{1} / / K\right) \text {. }
$$


Consider the two quantities

$$
\operatorname{tr} R_{\text {cusp }}\left(f^{G_{0}} * b_{E / F_{0}}(V)\right)
$$

and

$$
\operatorname{tr} R_{\text {cusp }}\left(f^{\mathrm{GL}_{2}} * b_{E / F}(V)\right)
$$

where $b_{E / ?}, ? \in\left\{F_{0}, F\right\}$ is the base change homomorphism defined on (a subalgebra of) $\mathcal{C}\left(G\left(\mathbb{A}_{F}\right)^{1}\right)$ in $\S 4$, and $f^{G_{0}} \in C_{c}^{\infty}\left(G_{0}\left(\mathbb{A}_{F}\right)^{1}\right), f^{\mathrm{GL}_{2}} \in C_{c}^{\infty}\left(\mathrm{GL}_{2}\left(\mathbb{A}_{F}\right)^{1}\right)$ are transfers of $f$ (the existence of transfers in this setting is proven in Lemma 4.2). Notice that by Theorem 1.1, the quantities (1.4.1) and (1.4.2) are given by (admittedly complicated) absolutely convergent sums and integrals that are geometric in nature. It is not hard to argue as in [11] that the equality of (1.4.1) and (1.4.2) for all $f$ implies that any $\mathrm{Gal}(E / F)$-invariant cuspidal automorphic representation of $G\left(\mathbb{A}_{F}\right)$ spherical at all places descends to $\mathrm{GL}_{2}\left(\mathbb{A}_{F}\right)$ (compare [11], Theorem 1.2).

Remark. The existence of base changes of cuspidal automorphic representations $\pi$ of $\mathrm{GL}_{2}\left(\mathbb{A}_{F}\right)^{1}$ spherical at all places would also follow if we knew somehow that $\operatorname{tr} \pi\left(b_{E / F}(V)\right) \neq 0$, but it is not clear how to prove this without knowing the existence of the base change.

We emphasize, once again, that (1.4.1) and (1.4.2) are built out of absolutely convergent geometric sums. This is not easy to come by in this subject. The primary difficulty in all cases where Langlands' beyond endoscopy proposal [18] has been carried out [26], [14], [27] has been obtaining an absolutely convergent sum out of limiting forms of trace formulae, and, to date, it has never been carried out to prove functoriality in a case that was not known by some other method. As explained in [24], this task is expected to become even more difficult in other situations. One motivation for proving Theorem 1.2 is that we can contemplate proving that (1.4.1) is equal to (1.4.2), and this might provide a way to move around the analytic difficulties of the beyond endoscopy proposal in certain cases.

\subsection{Outline}

In the following section, we give our conventions on Haar measures for the paper. In $\S 3$ we record some known results on Whittaker functions and prove the crucial result Corollary 3.5, which, combined with the Casselman-Shalika-Shintani formula, allows us to construct Hecke operators $\mathbb{W}_{g}$ whose Hecke eigenvalues are the values of Whittaker functions at $g \in G\left(\mathbb{A}_{F}\right)$. We then record what we mean by transfers of test functions in our setting in $\S 4$.

Finally, in $\S 5$ we construct $V$. The idea is to use the special Hecke operators $\mathbb{W}_{g}$ to build a weight consisting of the period of a cusp form over a unitary group into the trace formula. This weight isolates the representations of $G\left(\mathbb{A}_{F}\right)$ invariant under $\iota$ by work of Jacquet (we actually require the refinements of [6]). These $V$ are then used in $\S 5.2$ to prove our main result, Theorem 1.2.

\section{Haar measures}

Throughout this paper, we normalize Haar measures as in the following table, which is consistent with the normalizations in [7]: 


\begin{tabular}{|c|c|}
\hline group & normalization \\
\hline discrete & counting measure \\
\hline $\mathbb{R}$ & Lebesgue measure \\
\hline$i \mathbb{R}$ & through $x \mapsto i x$ \\
\hline$E_{w}, w$ archimedian & {$\left[E_{w}: \mathbb{R}\right]$ times the Lebesgue measure on $E_{w} \cong \mathbb{R}^{\left[E_{w}: \mathbb{R}\right]}$} \\
\hline$E_{w}, w$ nonarchimedian & $\operatorname{meas}\left(\mathcal{O}_{E_{w}}\right)=1$ \\
\hline $\mathbb{R}^{\times}, \mathbb{R}_{>0}$ & $d x^{\times}=\frac{d x}{|x|}$ \\
\hline$E_{w}^{\times}, w$ archimedian & $d x^{\times}=\frac{d x}{|x|}$ \\
\hline$E_{w}^{\times}, w$ nonarchimedian & $\operatorname{meas}\left(\mathcal{O}_{E_{w}}^{\times}\right)=1$ \\
\hline $\mathbb{A}_{E}^{\times}$ & product measure \\
\hline$\left(\mathbb{A}_{E}^{\times}\right)^{1}$ & compatible with $\mathbb{A}_{E}^{\times}=\left(\mathbb{A}_{E}^{\times}\right)^{1} \times \mathbb{R}_{>0}$ \\
\hline$Z_{\mathrm{GL}_{2}}(\mathbb{A})$ & through $t \mapsto\left({ }_{t}^{t}\right)$ \\
\hline$U\left(E_{w}\right), U\left(\mathbb{A}_{E}\right)$ & through $x \mapsto\left(\begin{array}{cc}1 & x \\
1\end{array}\right)$ \\
\hline$T\left(E_{w}\right), T\left(\mathbb{A}_{E}\right), T\left(\mathbb{A}_{E}\right)^{1}$ & through $\left(t_{1}, t_{2}\right) \mapsto\left(\begin{array}{ll}{ }^{t_{1}} & \\
{ }^{2}\end{array}\right)$ \\
\hline $\mathrm{GL}_{2}\left(\mathcal{O}_{E_{w}}\right), K_{w}(w$ archimedian $)$ & measure $=1$ \\
\hline $\mathrm{GL}_{2}\left(E_{w}\right), \mathrm{GL}_{2}\left(\mathbb{A}_{E}\right)$ & compatible with the Iwasawa decomposition \\
\hline $\mathrm{GL}_{2}\left(\mathbb{A}_{E}\right)^{1}$ & compatible with $\mathrm{GL}_{2}\left(\mathbb{A}_{E}\right)=\mathrm{GL}_{2}\left(\mathbb{A}_{E}\right)^{1} \times \mathbb{R}_{>0}$ \\
\hline
\end{tabular}

Here, $\left(\mathbb{A}_{E}^{\times}\right)^{1}=\operatorname{ker}(|\cdot|)$. We will also require a Haar measure on $H\left(\mathbb{A}_{F}\right)$ where $H$ is the group of $\S 5.1$, but for the purposes of this paper there is no need to specify it; we simply fix one.

\section{Whittaker functions}

In this section, we collect some generalities on Whittaker functions. The main results are corollaries 3.2 and 3.5, which construct Hecke operators whose Hecke eigenvalues are the values of Whittaker functions.

None of the results of this section use anything about the extension $E / F$, so for this section $E$ is an arbitrary number field. We let $K^{\infty}:=\mathrm{GL}_{2}\left(\widehat{\mathcal{O}}_{E}\right)$ and let $K_{\infty} \leq \mathrm{GL}_{2}\left(E_{\infty}\right)$ be the standard maximal compact subgroup.

\subsection{Generalities}

Let $\varphi$ be a smooth cuspidal function in $L^{2}\left(\mathrm{GL}_{2}(E) \backslash \mathrm{GL}_{2}\left(\mathbb{A}_{E}\right)^{1}\right)$ and let $\psi: E \backslash \mathbb{A}_{E} \longrightarrow \mathbb{C}^{\times}$ be the additive character

$$
\psi:=\psi_{\mathbb{Q}} \circ \operatorname{tr}_{\mathbb{A}_{E} / \mathbb{A}_{\mathbb{Q}}}
$$

where $\psi_{\mathbb{Q}}:=\psi_{\infty} \times \prod_{p} \psi_{p}$ with

$$
\psi_{\infty}(a):=e^{-2 \pi i a} \quad \text { and } \quad \psi_{p}(a):=e^{2 \pi i \operatorname{pr}(a)} .
$$

Here, the principal part $\operatorname{pr}(a) \in \mathbb{Z}\left[p^{-1}\right]$ is any element such that $a \equiv \operatorname{pr}(a)\left(\bmod \mathbb{Z}_{p}\right)$. We set

$$
\psi_{m}(x):=\psi(m x)
$$

for $m \in E$. As usual for each $m \in E$, we have Whittaker functions

$$
W_{m}^{\varphi}(g):=\int_{E \backslash \mathbb{A}_{E}} \varphi\left(\left(\begin{array}{ll}
1 & x \\
0 & 1
\end{array}\right) g\right) \psi(-m x) d x .
$$


To ease notation, we set

$$
W^{\varphi}:=W_{1}^{\varphi} .
$$

Since $\varphi$ is cuspidal, $W_{m}^{\varphi}(g)$ is identically zero as a function of $g$ if $m=0$. By taking Fourier expansions, we obtain the Whittaker expansion

$$
\varphi(g)=\frac{1}{\sqrt{d}_{E}} \sum_{m \in E^{\times}} W_{m}^{\varphi}(g) .
$$

Assume now that $\varphi$ is in the space $V_{\pi}$ of a cuspidal automorphic representation $\pi$ of $\mathrm{GL}_{2}\left(\mathbb{A}_{E}\right)$. Then $W_{m}^{\varphi}(g)$ is identically zero as a function of $g \in \mathrm{GL}_{2}\left(\mathbb{A}_{E}\right)$ if and only if $m=0$. For $m \in E^{\times}$, we obtain nonzero intertwining maps

$$
\begin{aligned}
V_{\pi} & \longrightarrow \mathcal{W}\left(\pi, \psi_{m}\right) \\
\varphi & \longmapsto W_{m}^{\varphi}
\end{aligned}
$$

to the Whittaker model $\mathcal{W}\left(\pi, \psi_{m}\right)$ of $\pi$ with respect to $\psi_{m}$. Here, $\mathcal{W}\left(\pi, \psi_{m}\right)$ and its local analogues are defined as in $([4] \S 1.2)$.

We recall that Whittaker functions of pure tensors $\varphi=\otimes_{w} \varphi_{w}$ factor into local Whittaker functions:

$$
W_{m}^{\varphi}(g)=\prod_{w} W_{m, w}^{\varphi_{w}}(g)
$$

where the product is over places $w$ of $E$ and $W_{m, w}^{\varphi_{w}} \in \mathcal{W}\left(\pi_{w}, \psi_{m w}\right)$, the Whittaker model of $\pi_{w}$ with respect to $\psi_{m w}$.

We note that for a smooth $\varphi$, a $\beta \in E^{\times}$, and $g \in \mathrm{GL}_{2}\left(\mathbb{A}_{E}\right)$, one has

$$
W_{\beta}^{\varphi}(g)=W^{\varphi}\left(\left(\begin{array}{ll}
{ }_{1} &
\end{array}\right) g\right)
$$

which follows from a change of variables in (3.1.3) together with the left $\mathrm{GL}_{2}(E)$ invariance of $\varphi$.

Finally, we record a definition used in the statement of Theorem 1.2 above. Let

$$
\delta \in \mathbb{A}_{E}^{\infty} \cap \widehat{\mathcal{O}}_{E}
$$

be an idele whose associated ideal is the absolute different $\mathcal{D}_{E}$ of $E$ over $\mathbb{Q}$. Let $\pi_{w}$ be a generic irreducible admissible representation of $\mathrm{GL}_{2}\left(E_{w}\right)$. We say that $W \in \mathcal{W}\left(\pi_{w}, \psi_{w}\right)$ is a Whittaker newform if the following conditions hold:

(1) It is $K_{w}$-finite.

(2) The identity

$$
|\delta|_{w}^{s-1 / 2} \int_{E_{w}^{\times}} W\left(\begin{array}{ll}
m & \\
&
\end{array}\right)|m|_{w}^{s-1 / 2} d m^{\times}=L\left(s, \pi_{w}\right)
$$

holds and the integral on the left is absolutely convergent for $\operatorname{Re}(s)$ large enough.

(3) If $w$ is archimedian, then $W$ has minimial $K_{w}$-type among all vectors $W^{\prime} \in \mathcal{W}\left(\pi_{w}, \psi_{w}\right)$ such that (1) and (2) hold

(4) If $w$ is nonarchimedian, then $W$ is a newform.

The factor $|\delta|_{w}^{s-1 / 2}$ occurs here because we have chosen our standard character $\psi_{w}$ to have conductor $\mathcal{D}_{E w}$. If $\varphi$ is a smooth function in the space of a cuspidal automorphic representation $\pi$ realized in the cuspidal subspace of $L^{2}\left(G L_{2}(E) \backslash G L_{2}\left(\mathbb{A}_{E}\right)^{1}\right)$, then we say that $\varphi$ is a Whittaker newform if it is a pure tensor $\left(\varphi=\otimes_{w} \varphi_{w}\right)$ and $W^{\varphi}$ can be factored as $W^{\varphi}=\prod_{w} W_{w}^{\varphi_{w}}$ where $W_{w}^{\varphi_{w}}$ is a Whittaker newform for all $w$. 
Whittaker newforms always exist. For the archimedian case see [23]. In the nonarchimedian case, the Whittaker newform is a choice of normalization of a newform (or new vector) in the usual sense (see [10], Proposition 6.17 or [25], § 2, Summary).

\subsection{The Casselman-Shalika-Shintani formula}

For the remainder of this section, we work in a local setting. Thus, we fix a place $w$ and omit it from notation, writing $E:=E_{w}$.

Assume for this subsection that $w$ is nonarchimedian. Let $m \in E^{\times} \cap \mathcal{O}_{E}$. We let

$$
\mathbb{1}_{m}(g):=\mathbb{1}_{\left\{x \in M_{2}\left(\mathcal{O}_{E}\right): \operatorname{det}(x) \mathcal{O}_{E}=m \mathcal{O}_{E}\right\}}(g) \in C_{c}^{\infty}\left(\mathrm{GL}_{2}(E) / / K\right) .
$$

Here and below, unless otherwise specified, $\mathbb{1}_{X}$ is the characteristic function of a set $X$.

The following is the Casselman-Shalika formula, due in the case of $\mathrm{GL}_{n}$ to Shintani:

Theorem 3.1. Let $\pi$ be a generic unramified representation of $\mathrm{GL}_{2}(E)$. If $W \in \mathcal{W}(\pi, \psi)$ is fixed by $K$ then for $m \in E^{\times} \cap \mathcal{O}_{E}$, one has

$$
\frac{W\left(\begin{array}{c}
m / \delta \\
1
\end{array}\right)}{|m| W\left(\begin{array}{c}
1 / \delta \\
\end{array}{ }_{1}\right)}=\operatorname{tr} \pi\left(\mathbb{1}_{m}\right)
$$

Here, $\delta$ is as in (3.1.7).

Proof. This is well known. One possible reference is [16] (Proposition 4.6), bearing in mind that the adelic computation contained therein could just as easily be done locally.

Let $B \leq \mathrm{GL}_{2}$ denote the Borel subgroup of upper-triangular matrices. For

$$
\left(\begin{array}{c}
1 \\
1 \\
1
\end{array}\right)\left({ }^{z m / \delta}{ }_{z}\right) k \in B(E) K=\mathrm{GL}_{2}(E)
$$

(with $k \in K$ ) let

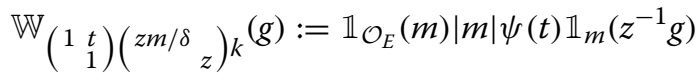

where $\psi$ is as in (3.1.1). We have the following corollary of Theorem 3.1:

Corollary 3.2. Let $\pi$ be a generic unramified representation of $\mathrm{GL}_{2}(E)$. If $W \in \mathcal{W}(\pi, \psi)$ is a Whittaker newform and $g \in \mathrm{GL}_{2}(E)$ then

$$
\pi\left(\mathbb{W}_{g}\right) W=W(g) W .
$$

Proof. Let $\omega$ denote the central character of $\pi$. One has

$$
\begin{aligned}
& \mathbb{W}\left(\begin{array}{cc}
1 & t \\
1
\end{array}\right)\left(z m / \delta_{z}\right) k=\psi(t) \mathbb{W}\left({ }_{z m / \delta}{ }_{z}\right) \text { and } \\
& W\left(\left(\begin{array}{cc}
1 & t \\
1
\end{array}\right)\left({ }^{z m / \delta}{ }_{z}\right) k\right)=\psi(t) W\left(\begin{array}{l}
z m / \delta \\
z
\end{array}\right)=\psi(t) \omega(z) W\left(\begin{array}{c}
m / \delta \\
\end{array}{ }_{1}\right) .
\end{aligned}
$$

Moreover,

$$
\left.\pi\left(\mathbb{W}_{(z m / \delta}\right)\right) W=\omega(z) \pi\left(\mathbb{W}\left(m_{z} / \delta_{1}\right)\right) W
$$

so it suffices to verify the corollary for $g=\left({ }^{m / \delta}{ }_{1}\right)$. For this, we recall that if $W \in \mathcal{W}(\pi, \psi)$ is a Whittaker newform then $W\left(\begin{array}{c}m / \delta \\ 1\end{array}\right) \neq 0$ only if $m \in \mathcal{O}_{E}$. One reference for this fact is 
the summary of [25] ( $\S 2)$, bearing in mind that in loc. cit. the character $\psi$ is normalized to have conductor $\mathcal{O}_{E}$, whereas we normalized our $\psi$ to have conductor $\mathcal{D}_{E}$. Thus, in particular, we can and do assume that $m \in \mathcal{O}_{E}-0$. To handle this final case, we use the fact that $\pi\left(\mathbb{1}_{m}\right)$ acts via the scalar $\operatorname{tr} \pi\left(\mathbb{1}_{m}\right)$ on $W$, Theorem 3.1, and the fact that $W\left(\begin{array}{ll}1 / \delta & \end{array}\right)=1([25], \S 2$, Summary) to deduce the corollary.

\subsection{A replacement for Theorem 3.1 in the archimedian case}

Assume that $E=E_{w}$ is an archimedian local field. In this section, we construct test functions in

$$
\mathcal{C}\left(\mathrm{GL}_{2}(E)\right)
$$

which is defined to be the Frechet space of smooth functions $f$ on $\mathrm{GL}_{2}(E)$ such that for all

$$
X, Y \in U\left(\operatorname{Res}_{E / \mathbb{R}} \mathfrak{g l}_{2 E} \otimes_{\mathbb{R}} \mathbb{C}\right)
$$

one has

$$
\|X * f * Y\|_{1}:=\int_{\mathrm{GL}_{2}(E)}|X * f * Y| d g<\infty .
$$

In order to construct these functions, we recall the explicit description of the standard model of an induced irreducible admissible representation of $\mathrm{GL}_{2}(E)$. The basic reference for the constructions is [15] ( $\$ 5-6)$. There is a brief summary in $[28](\S 2.2)$.

Let $\left(\pi, V_{\pi}\right)$ be an irreducible representation of $\mathrm{GL}_{2}(E)$ with central character $\chi$. Consider the space $\mathcal{B}\left(\mu \chi, \mu^{-1}\right)$ of $K$-finite functions $\varphi \in C^{\infty}\left(\mathrm{GL}_{2}(E)\right)$ satisfying

$$
\varphi\left(z\left(\begin{array}{c}
y * \\
1
\end{array}\right) g\right)=\chi(z) \mu \chi(y)|y|^{1 / 2} \varphi(g) \text { for }(y, z, g) \in E^{\times} \times E^{\times} \times \mathrm{GL}_{2}(E)
$$

where $\mu: E^{\times} \longrightarrow \mathbb{C}^{\times}$is a quasicharacter. Letting $\mathfrak{g}$ denote the complexification of the Lie algebra of $\mathrm{GL}_{2}(E)$, one has that $\mathcal{B}\left(\mu \chi, \mu^{-1}\right)$ is a $(\mathfrak{g}, K)$-module, and upon replacing $\mu$ with $\chi^{-1} \mu^{-1}$ if necessary we can and do assume that $\pi$ (as a $(\mathfrak{g}, K)$-module) is equivalent to a subrepresentation (not merely a subquotient) of $\mathcal{B}\left(\mu \chi, \mu^{-1}\right)$. The space $\mathcal{B}\left(\mu \chi, \mu^{-1}\right)$ consists of vectors of the form

$$
\varphi_{\Phi}(g):=\mu \chi(\operatorname{det} g)|\operatorname{det} g|^{1 / 2} \int_{E^{\times}} \Phi((0, t) g) \mu^{2} \chi(t)|t| d t^{\times}
$$

for $\Phi$ in the Schwarz space $\mathcal{S}(E \times E)$.

For $g \in M_{2}(E)$, let

$$
\mathcal{F}_{P}(g):=4^{[E: \mathbb{R}]-1}(2 \pi)^{2(1-[E: \mathbb{R}])} P(g) e^{-\pi[E: \mathbb{R}] \operatorname{tr} g^{*} g}|\operatorname{det} g|^{\frac{1}{2}}
$$

where $P(g)$ is a polynomial in the entries of $g$ and their complex conjugates and $g^{*}:=\bar{g}^{t}$, the bar denoting complex conjugation (which is trivial if $E$ is real). It is clear that $\mathcal{F}_{P}$ restricts to a smooth function on $\mathrm{GL}_{2}(E)$.

For real numbers $r \in \mathbb{R}_{>0}$, let $\delta_{r}$ denote the distribution on $\mathrm{GL}_{2}(E)$ given on test functions $f \in C_{c}^{\infty}\left(\mathrm{GL}_{2}(E)\right)$ by

$$
\delta_{r}(f):=\int_{\mathrm{GL}_{2}(E)^{1}} f\left(r g^{1}\right) d g^{1}
$$

where

$$
\mathrm{GL}_{2}(E)^{1}:=\operatorname{ker}\left(|\cdot| \circ \operatorname{det}: \mathrm{GL}_{2}(E) \longrightarrow \mathbb{R}_{>0}\right)
$$


and $d g^{1}$ is chosen to be compatible with the decomposition

$$
\mathrm{GL}_{2}(E)=\mathrm{GL}_{2}(E)^{1} \times \mathbb{R}_{>0}
$$

and the choices of measures in $\S 2$. Less formally, $\delta_{r}$ is the Dirac distribution associated to the $\mathrm{GL}_{2}(E)^{1}$-homogeneous submanifold

$$
\left\{g \in \mathrm{GL}_{2}(E):|\operatorname{det} g|=r^{2[E: \mathbb{R}]}\right\}
$$

of $\mathrm{GL}_{2}(E)$.

Assume that the representation

$$
K \times K \longrightarrow \operatorname{Aut}\left\langle x \mapsto \mathcal{F}_{P}\left(k_{1}^{-1} x k_{2}\right): k_{1}, k_{2} \in K\right\rangle
$$

is finite-dimensional and equivalent to an irreducible representation of the form $\rho \otimes \rho^{\vee}$ of $K \times K$, where $\rho$ is an irreducible representation of $K$.

Lemma 3.3. Assume that $\pi$ is an irreducible unitary representation of $\mathrm{GL}_{2}(E)$. One has a well-defined operator

$$
\pi\left(\mathcal{F}_{P} \delta_{r}\right)
$$

on the space of $\pi$, bounded with respect to the given $L^{2}$-norm. Let $\varphi$ be a vector in $\pi$, viewed as a subrepresentation of $\mathcal{B}\left(\mu \chi, \mu^{-1}\right)$. We assume that $\varphi$ has $K$-type $\rho^{\prime}$, where $\rho^{\prime}$ is irreducible. If $\rho \neq \rho^{\prime}$, then $\pi\left(\mathcal{F}_{P} \delta_{r}\right) \varphi=0$. If $\rho=\rho^{\prime}$ is trivial then $\pi\left(\mathcal{F}_{P} \delta_{r}\right)$ acts via the scalar

$$
\int_{E^{\times} \times E} \mathcal{F}_{P}\left(\begin{array}{cc}
\frac{r}{|y|^{1 /(2[E: Q])} y} & x \\
& \frac{r}{|y|^{1 /(2[E: \mathbb{R}])}}
\end{array}\right) \chi\left(r /|y|^{1 /(2[E: \mathbb{R}])}\right) \mu \chi(y) \frac{d y^{\times}}{|r|} d x
$$

on $\varphi$. Moreover, the integral in this expression is absolutely convergent.

Proof. To prove that the operator is bounded, we proceed as in [12] (§ 1.1). First, it suffices to prove that the restriction of $\pi\left(\mathcal{F}_{P} \delta_{r}\right)$ to smooth vectors is well defined and bounded. Since $\pi$ is unitary, it is enough to show that the $L^{1}$ norm of (the distribution) $\mathcal{F}_{P} \delta_{r}$ is finite. Let $\mathcal{G}_{1} \in \mathcal{S}\left(M_{2}(E)\right.$ ) be a nonnegative Schwartz function (such as $\mathcal{F}_{P}$ ). Then there are nonnegative Schwartz functions $\mathcal{G}_{2} \in \mathcal{S}\left(M_{2}(E)\right)$ and $\mathcal{G}_{3} \in \mathcal{S}(E \times E)$ such that

$$
\begin{aligned}
& \int_{\mathrm{GL}_{2}(E)^{1}} \mathcal{G}_{1}\left(r g^{1}\right) d g^{1} \\
& =\int_{E^{\times} \times E \times K} \mathcal{G}_{1}\left(\frac{r}{|y|^{1 /(2[E: \mathbb{R}])}}\left(\begin{array}{cc}
y & x \\
& 1
\end{array}\right) k\right) \frac{d y^{\times}}{|y|} d x d k \\
& =\int_{E^{\times} \times E} \mathcal{G}_{2}\left(\frac{r}{|y|^{1 /(2[E: \mathbb{R}])}}\left(\begin{array}{cc}
y & x \\
& 1
\end{array}\right)\right) \frac{d y^{\times}}{|y|} d x \\
& =\int_{E^{\times} \times E} \mathcal{G}_{2}\left(\begin{array}{cc}
\frac{r}{|y|^{1 /(2[E: \mathbb{R}])} y} & x \\
& \frac{r}{|y|^{1 /(2[E: \mathbb{R}])}}
\end{array}\right) \frac{d y^{\times}}{|y|^{1 / 2} r^{[E: \mathbb{R}]}} d x \\
& =\int_{E^{\times}} \mathcal{G}_{3}\left(\frac{r}{|y|^{1 /(2[E: \mathbb{R}])}} y, \frac{r}{|y|^{1 /(2[E: \mathbb{R}])}}\right) \frac{d y^{\times}}{|y|^{1 / 2} r^{[E: \mathbb{R}]}}<\infty .
\end{aligned}
$$

It is not hard to see that this computation implies that the $L^{1}$-norm of $\mathcal{F}_{P} \delta_{r}$ (as a distribution) is finite, and this completes the proof of boundedness.

If $\varphi$ does not lie in the $\rho$-isotypic subspace of $\pi$, then the assertion of the lemma is clear. Suppose therefore that $\varphi \neq 0$ does lie in the $\rho$-isotypic subspace (which implies in 
particular that this subspace is nonempty). We recall that $\rho$ occurs with multiplicity at most 1 in $\pi$ ([15], § 5, Lemma 6.1). Thus, our assumptions on the $K$-type of $\mathcal{F}_{P}$ and Schur's lemma imply that the operator $\pi\left(\mathcal{F}_{P} \delta_{r}\right)$ acts via a scalar on the $\rho$-isotypic subspace. We must compute this scalar.

Choose $\Phi \in \mathcal{S}(E \times E)$ such that $\varphi_{\Phi}$ is in the $\rho$-isotypic subspace of $\pi$ and

$$
\varphi_{\Phi}\left(\begin{array}{ll}
1 & \\
&
\end{array}\right) \neq 0 \text {. }
$$

That this is always possible can be seen by realizing that the integral defining $\varphi_{\Phi}$ is a Tate integral, compare ([15], Lemma 5.13.1 and Lemma 6.3.1).

Using the Iwasawa decomposition, we compute

$$
\begin{aligned}
\left(\pi\left(\mathcal{F}_{P} \delta_{r}\right) \varphi_{\Phi}\right)\left({ }_{1}{ }_{1}\right) & =\int_{\mathrm{GL}_{2}(E)^{1}} \mathcal{F}_{P}\left(r g^{1}\right) \varphi_{\Phi}\left(r g^{1}\right) d g^{1} \\
& =\int_{E^{\times} \times E \times K} \mathcal{F}_{P}\left(\frac{r}{|y|^{1 /(2[E: \mathbb{R}])}}\left(\begin{array}{cc}
y & x \\
1
\end{array}\right) k\right) \varphi_{\Phi}\left(\frac{r}{|y|^{1 /(2[E: \mathbb{R}])}}\left(\begin{array}{rr}
y & x \\
1
\end{array}\right) k\right) \frac{d y^{\times}}{|y|} d x d k \\
& =\int_{E^{\times} \times E} \mathcal{F}_{P}\left(\frac{r}{|y|^{1 /(2[E: \mathbb{R}])}}\left(\begin{array}{rr}
y & x \\
& 1
\end{array}\right)\right) \varphi_{\Phi}\left(\frac{r}{|y|^{1 /(2[E: \mathbb{R}])}}\left(\begin{array}{rr}
y & x \\
1
\end{array}\right)\right) \frac{d y^{\times}}{|y|} d x
\end{aligned}
$$

(see $\S 2$ for normalizations of measures).

Substituting the definition (3.3.5) of $\varphi_{\Phi}$, we obtain

$$
\begin{aligned}
& \int_{E^{\times}} \Phi(0, t) \mu^{2} \chi(t)|t| d t^{\times} \int_{E^{\times} \times E} \mathcal{F}_{P}\left(\frac{r}{|y|^{1 /(2[E: \mathbb{R}])}}\left(\begin{array}{cc}
y & x \\
1
\end{array}\right)\right) \mu \chi(y) \chi\left(r /|y|^{1 /(2[E: \mathbb{R}])}\right) \frac{d y^{\times}}{|y|^{1 / 2}} d x \\
& =\varphi_{\Phi}\left({ }^{1}{ }_{1}\right) \int_{E^{\times} \times E} \mathcal{F}_{P}\left(\begin{array}{cc}
\frac{r}{|y|^{1 /(2[E: \mathbb{R}])} y} & \frac{x}{|y|^{1 /(2[E: \mathbb{R})}}
\end{array}\right) \chi\left(r /|y|^{1 /(2[E: \mathbb{R}])}\right) \mu \chi(y) \frac{d y^{\times}}{|r|} d x .
\end{aligned}
$$

By (3.3.8), we conclude that the scalar by which $\pi\left(\mathcal{F}_{P} \delta_{r}\right)$ acts on the $\rho$-isotypic subspace is

$$
\int_{E^{\times} \times E} \mathcal{F}_{P}\left(\begin{array}{cc}
\frac{r}{|y|^{1 /(2[E: \mathbb{R})} y} & x \\
& \frac{r}{|y|^{1 / 2[E: \mathbb{R}])}}
\end{array}\right) \chi\left(r /|y|^{1 /(2[E: \mathbb{R}])}\right) \mu \chi(y) \frac{d y^{\times}}{|r|} d x .
$$

For the remainder of this subsection assume that $\pi$ is spherical. For each $h=$ $\left(\begin{array}{c}1 \\ 1\end{array}\right)\left(\begin{array}{c}z m \\ z\end{array}\right) k \in \mathrm{GL}_{2}(E)$ with $k \in K$ define a distribution $\mathbb{W}_{h}$ on $\mathrm{GL}_{2}(E)$ by setting

$$
\mathbb{W}\left(\begin{array}{cc}
1 & t \\
1
\end{array}\right)\left({ }^{z m}{ }_{z}\right) k(g)
$$

equal to

$$
\frac{\psi(t)|m|^{1 / 2}}{2} \mathcal{F}_{1} \delta_{|m|^{1 /(2[E: \mathbb{R}])}}\left(z^{-1} g\right) .
$$

Lemma 3.4. For any $\Psi \in C_{c}^{\infty}\left(\mathrm{GL}_{2}(E)\right)$, one has $\Psi * \mathbb{W}_{g} \in \mathcal{C}\left(\mathrm{GL}_{2}(E)\right)$. In fact, for any $A \in \mathbb{R}_{>0}$ and

$$
X, Y \in U\left(\operatorname{Res}_{E / \mathbb{R}} \mathfrak{g l}_{2 E} \otimes_{\mathbb{R}} \mathbb{C}\right)
$$

one has

$$
\left\|X * \Psi * \mathbb{W}\left(m_{1}\right) * Y\right\|_{1} \ll \Psi, A, X, Y|m|^{-A}
$$

for $|m|>1$ and there exists a $B>0$ such that

$$
\left\|X * \Psi * \mathbb{W}_{\left(m_{1}\right)} * Y\right\|_{1} \ll \Psi, B, X, Y|m|^{-B}
$$

for $|m| \leq 1$. 
Proof. It clearly suffices to prove the two bounds.

As explained in [12] (§ 1.8, p. 115-116), we have

$$
X * \mathcal{F}_{1} * Y=\mathcal{F}_{P}
$$

for some $P$. Moreover, any operator in the subalgebra

$$
U\left(\operatorname{Res}_{E / \mathbb{R}} \mathfrak{s l}_{2 E} \otimes_{\mathbb{R}} \mathbb{C}\right) \leq U\left(\operatorname{Res}_{E / \mathbb{R}} \mathfrak{g l}_{2 E} \otimes_{\mathbb{R}} \mathbb{C}\right)
$$

is insensitive to the difference between $\mathcal{F}_{P}$ and $\mathcal{F}_{P} \delta_{|m|}$, and the algebra on the right is generated (as an algebra) by these elements together with central operators corresponding to the Lie algebra of $Z_{\operatorname{Res}_{E / \mathbb{R}} \mathrm{GL}_{2}}$. These central operators can be absorbed into $\Psi$. It therefore suffices to show that for $\Psi \in C_{c}^{\infty}\left(\mathrm{GL}_{2}(E)\right)$ and polynomials $P$ as above, we have

$$
\begin{aligned}
& \left\|\Psi * \mathcal{F}_{P} \delta_{r}\right\|_{1} \ll_{\Psi, A, P} r^{-A} \text { for } r>1 \\
& \left\|\Psi * \mathcal{F}_{P} \delta_{r}\right\|_{1} \ll \Psi, B, P \\
& r^{-B} \text { for } r \leq 1 .
\end{aligned}
$$

for any $A>0$ and some $B>0$. But

$$
\begin{aligned}
\left\|\Psi * \mathcal{F}_{P} \delta_{r}\right\|_{1} & =\int_{\mathrm{GL}_{2}(E)}\left|\int_{\mathrm{GL}_{2}(E)^{1}} \Psi\left(x\left(r g^{1}\right)^{-1}\right) \mathcal{F}_{P}\left(r g^{1}\right) d g^{1}\right| d x \\
& \leq \int_{\mathrm{GL}_{2}(E)} \int_{\mathrm{GL}_{2}(E)^{1}}\left|\Psi\left(x\left(r g^{1}\right)^{-1}\right) \mathcal{F}_{P}\left(r g^{1}\right)\right| d g^{1} d x \\
& =\int_{\mathrm{GL}_{2}(E)} \int_{\mathrm{GL}_{2}(E)^{1}}\left|\Psi(x) \mathcal{F}_{P}\left(r g^{1}\right)\right| d g^{1} d x \\
& \ll \Psi \int_{\mathrm{GL}_{2}(E)^{1}}\left|\mathcal{F}_{P}\left(r g^{1}\right)\right| d g^{1} .
\end{aligned}
$$

As in (3.3.7), this is bounded by

$$
\int_{E^{\times}} \mathcal{G}_{3}\left(\frac{r}{|y|^{1 /(2[E: \mathbb{R}])}} y, \frac{r}{|y|^{1 /(2[E: \mathbb{R}])}}\right) \frac{d y^{\times}}{|y|^{1 / 2} r^{[E: \mathbb{R}]}}
$$

for some $\mathcal{G}_{3} \in \mathcal{S}(E \times E)$. Assume now that $r>1$. The contribution of $|y| \leq 1$ to this integral is rapidly decreasing as a function of $r \longrightarrow \infty$ by consideration of the second variable. The contribution of $|y| \geq 1$ is as well by consideration of the first variable. Thus, we obtain the first bounds claimed in (3.3.11).

Assume now that $r \leq 1$. For the contribution of $|y| \leq 1$, we obtain a bound of a constant depending on $A>[E: \mathbb{R}]$ times

$$
\begin{aligned}
& \int_{y \in E^{\times}:|y| \leq 1} \max \left(1, \frac{r}{|y|^{1 /(2[E: \mathbb{R}])}}\right)^{-A} \frac{d y^{\times}}{|y|^{1 / 2} r^{[E: \mathbb{R}]}} \\
& \ll \int_{0 \leq t \leq r^{2}}\left(\frac{r}{\sqrt{t}}\right)^{-A} \frac{d t^{\times}}{(\sqrt{t} r)^{[E: \mathbb{R}]}}+\int_{r^{2} \leq t \leq 1} \frac{d t^{\times}}{(\sqrt{t} r)^{[E: \mathbb{R}]}} \\
& \ll r^{-2[E: \mathbb{R}]} .
\end{aligned}
$$

As for the contribution of $|y| \geq 1$, for any $A>[E: \mathbb{R}]$, we obtain a bound of a constant depending on $A$ times

$$
\begin{aligned}
& \int_{y \in E^{\times}:|y| \geq 1} \max \left(1, \frac{r y}{|y|^{1 /(2[E: \mathbb{R}])}}\right)^{-A} \frac{d y^{\times}}{|y|^{1 / 2} r^{[E: \mathbb{R}]}} \\
& \ll \int_{1 \leq t \leq r^{-2}} \frac{d t^{\times}}{(\sqrt{t} r)^{[E: \mathbb{R}]}}+\int_{t \geq r^{-2}}(r \sqrt{t})^{-A} \frac{d t^{\times}}{(\sqrt{t} r)^{[E: \mathbb{R}]}} \\
& \ll r^{-2[E: \mathbb{R}]} .
\end{aligned}
$$


These two bounds yield the second assertion in (3.3.11).

The reason for the notation $\mathbb{W}_{g}$ is apparent given the following Corollary:

Corollary 3.5. Let $\pi$ be unitary and spherical, and let $W \in \mathcal{W}(\pi, \psi)$ be the spherical Whittaker newform. Then $\pi\left(\mathbb{W}_{g}\right)$ annihilates the orthogonal complement of the line spanned by $W$, and acts via the scalar $W(g)$ on the line spanned by $W$.

Proof. It is enough to verify the corollary in the special case where $g=\left(\begin{array}{ll}m & \\ & 1\end{array}\right)$ (compare the proof of Corollary 3.2). By Lemma 3.3, it suffices to show that

$$
\operatorname{tr} \pi\left(\mathbb{W}\left(\begin{array}{ll}
r_{1} &
\end{array}\right)=W\left(\begin{array}{ll}
r & \\
& 1
\end{array}\right)\right.
$$

for $r \in \mathbb{R}_{>0}$. Both sides of this expression are smooth functions of $r$. It is well known that the right hand side is rapidly decreasing as $r \longrightarrow \infty$ and of order at most $r^{-B}$ for some $B>0$ as $r \longrightarrow 0$ (see the proofs of [15] (Theorem 5.13 and Theorem 6.3)) and the same is true of the left hand side by Lemma 3.4. Thus, to prove that the two are equal it is enough to verify that their Mellin transforms are equal. Recall (see $\S 2$ ) that our choice of Haar measure on $E$ is the Lebesgue measure if $E$ is real and twice the Lebesgue measure (on $\mathbb{R}^{2}$ ) if $E$ is complex. Thus, essentially by definition, for $\operatorname{Re}(s)$, sufficiently large one has

$$
2^{[E: \mathbb{R}]} \pi^{[E: \mathbb{R}]-1} \int_{\mathbb{R}_{>0}} W\left({ }^{r}{ }_{1}\right) r^{[E: \mathbb{R}]\left(s-\frac{1}{2}\right)} \frac{d r}{r}=L(s, \pi) .
$$

On the other hand, by Lemma 3.3 for $\operatorname{Re}(s)$, sufficiently large

$$
\begin{aligned}
& 2^{[E: \mathbb{R}]} \pi^{[E: \mathbb{R}]-1} \int_{\mathbb{R}_{>0}} \operatorname{tr} \pi\left(\mathbb{W}\left({ }^{r}{ }_{1}\right)\right) r^{[E: \mathbb{R}]\left(s-\frac{1}{2}\right)} d r^{\times} \\
& =2^{[E: \mathbb{R}]-1} \pi^{[E: \mathbb{R}]-1} \int_{\mathbb{R}_{>0}} \int_{E^{\times} \times E} \mathcal{F}_{1}\left(\begin{array}{cc}
\left(\frac{r}{|y|^{1 /[E: \mathbb{R}]}}\right)^{1 / 2} y & x \\
& \left(\frac{r}{|y|^{1 /[E: \mathbb{R}]}}\right)^{1 / 2}
\end{array}\right) \\
& \times \chi\left(\left(\frac{r}{|y|^{1 /[E: \mathbb{R}]}}\right)^{1 / 2}\right) \mu \chi(y) d y^{\times} r^{[E: \mathbb{R}]\left(s-\frac{1}{2}\right)} d r^{\times} d x \\
& =2^{[E: \mathbb{R}]-1} \pi^{[E: \mathbb{R}]-1} \int_{\mathbb{R}_{>0}} \int_{E^{\times} \times E} \mathcal{F}_{1}\left(\begin{array}{cc}
\sqrt{r} y & x \\
& \sqrt{r}
\end{array}\right) \chi(\sqrt{r}) \mu \chi(y)|y r|^{s-\frac{1}{2}} d y^{\times} d r^{\times} d x \\
& =\int_{E^{\times}} \int_{E^{\times} \times E} \mathcal{F}_{1}\left(\begin{array}{rr}
z y & x \\
& z
\end{array}\right) \chi(z) \mu \chi(y)\left|y z^{2}\right|^{s-\frac{1}{2}} d y^{\times} d z^{\times} d x \\
& =\int_{E^{\times} \times E^{\times}} \int_{E} \mathcal{F}_{1}(\underset{z}{y} \underset{z}{x}) d x|y z|^{s-1 / 2} \mu \chi(y) \mu^{-1}(z) d y^{\times} d z^{\times} \\
& =4^{[E: \mathbb{R}]-1}(2 \pi)^{2(1-[E: \mathbb{R}])} \int_{E^{\times} \times E^{\times}} \int_{E} e^{-[E: \mathbb{R}] \pi(x \bar{x}+y \bar{y}+z \bar{z})} d x|y z|^{s} \mu \chi(y) \mu^{-1}(z) d y^{\times} d z^{\times} \\
& =4^{[E: \mathbb{R}]-1}(2 \pi)^{2(1-[E: \mathbb{R}])} \int_{E^{\times} \times E^{\times}} e^{-[E: \mathbb{R}] \pi(y \bar{y}+z \bar{z})}|y z|^{s} \mu \chi(y) \mu^{-1}(z) d y^{\times} d z^{\times} \\
& =L(s, \mu \chi) L\left(s, \mu^{-1}\right)=L(s, \pi) \text {. }
\end{aligned}
$$




\section{Test functions and transfer}

For this section, we let $E / F$ be a finite extension of number fields (not necessarily Galois) that is everywhere unramified and split at all infinite places. Let $G:=\operatorname{Res}_{\mathcal{O}_{E} / \mathcal{O}_{F}} \mathrm{GL}_{2}$, let $K_{\infty} \leq G\left(F_{\infty}\right)$ be the standard maximal compact subgroup and let $K^{\infty} \leq G\left(\mathbb{A}_{F}^{\infty}\right)$ be a compact open subgroup.

Consider the Frechet space

$$
\mathcal{C}\left(G\left(\mathbb{A}_{F}\right)^{1}\right):=\mathcal{C}\left(G\left(\mathbb{A}_{F}\right)^{1}, K^{\infty}\right) .
$$

It consists of those smooth functions $f$ on $G\left(\mathbb{A}_{F}\right)$ that are right invariant under $K^{\infty}$ and satisfy

$$
\|X * f * Y\|_{L^{1}\left(G\left(\mathbb{A}_{F}\right)^{1}\right)}<\infty
$$

for all $X, Y \in U(\mathfrak{g})$, where

$$
\mathfrak{g}:=\operatorname{Lie}\left(G\left(F \otimes_{\mathbb{Q}} \mathbb{R}\right)\right) \otimes_{\mathbb{R}} \mathbb{C} .
$$

We also have the analogous Frechet space $\mathcal{C}\left(G\left(F_{\infty}\right)\right)$ at infinity; it consists of elements of $C^{\infty}\left(G\left(F_{\infty}\right)\right)$ such that $(4.0 .2)$ is valid for all $X, Y$ with $L^{1}\left(G\left(\mathbb{A}_{F}\right)^{1}\right)$ replaced by $L^{1}\left(G\left(F_{\infty}\right)\right)$. Note that this is not the same as the Harish-Chandra Schwartz space, whose elements may not be integrable.

The spaces

$$
\mathcal{C}\left(\mathrm{GL}_{2}\left(\mathbb{A}_{F}\right)^{1}\right):=\mathcal{C}\left(\mathrm{GL}_{2}\left(\mathbb{A}_{F}\right)^{1}, K^{\infty} \cap \mathrm{GL}_{2}\left(\mathbb{A}_{F}^{\infty}\right)\right) \text { and } \mathcal{C}\left(\mathrm{GL}_{2}\left(F_{\infty}\right)\right)
$$

are defined similarly. We note that these spaces are algebras under convolution. Moreover, if $\Pi$ is a cuspidal automorphic representation of $G\left(\mathbb{A}_{F}\right)^{1}$, then

$$
\operatorname{tr} \Pi: \mathcal{C}\left(G\left(\mathbb{A}_{F}\right)^{1}\right) \longrightarrow \mathbb{C}
$$

is a continuous linear functional (this is part of the main result of [7], for example, see Theorem 1.1 above). The assumption that $\Pi$ is cuspidal can be weakened somewhat, but it is not true for an arbitrary admissible representation.

Let $v$ be a place of $F$. We say that $\Psi \in C_{c}^{\infty}\left(\mathrm{GL}_{2}\left(F_{v}\right)\right)$ is a transfer of $f \in C_{c}^{\infty}\left(G\left(F_{v}\right)\right)$ if

$$
\operatorname{tr} \pi(\Psi)=\operatorname{tr} \Pi(f)
$$

is valid for all irreducible admissible representations $\pi$ of $\mathrm{GL}_{2}\left(F_{v}\right)$ with base change $\Pi$ to $G\left(F_{v}\right)$ (the notion of base change in this context is recalled in [11] $(\S 1)$ ).

Remarks. (1) This notion does not coincide with the usual notion of transfer in the theory of cyclic base change. (2) It would be interesting to define transfers of arbitrary functions in $\mathcal{C}\left(G\left(\mathbb{A}_{F}\right)^{1}\right)$, but we will not attempt this. Instead, we will make do with something weaker (see Lemma 4.2 below).

In this paper, we need to make explicit our choices of transfers. For the remainder of this section, let

$$
K^{\infty}:=G\left(\widehat{\mathcal{O}}_{F}\right)
$$

and let $K_{F}:=K \cap \mathrm{GL}_{2}\left(\mathbb{A}_{F}\right)$. We will define a continuous algebra homomorphism

$$
b_{E / F}: \mathcal{C}\left(G\left(\mathbb{A}_{F}\right)^{1} / / K\right) \longrightarrow \mathcal{C}\left(\mathrm{GL}_{2}\left(\mathbb{A}_{F}\right)^{1} / / K_{F}\right)
$$


(see Lemma 4.2 below). Here, the double slash indicates functions invariant on the left and right under $K$ (resp. $K_{F}$ ). The analogous convention will be used below.

First recall that for finite places $v$ of $F$ the base change maps

$$
b_{E / F}: C_{c}^{\infty}\left(G\left(F_{v}\right) / / K_{v}\right) \longrightarrow C_{c}^{\infty}\left(\mathrm{GL}_{2}\left(F_{v}\right) / / \mathrm{GL}_{2}\left(\mathcal{O}_{F_{v}}\right)\right)
$$

provide examples of transfers in the sense that $b_{E / F}(f)$ is a transfer of $f$ (compare [11], $\S$ 3.2).

Second, recall that $E / F$ is assumed to be split at all infinite places. We can therefore define a map

$$
b_{E / F}: \mathcal{C}\left(G\left(F_{\infty}\right) / / K_{\infty}\right) \longrightarrow \mathcal{C}\left(G_{2}\left(F_{\infty}\right) / / K_{F \infty}\right)
$$

by choosing an identification

$$
G\left(F_{\infty}\right)=\mathrm{GL}_{2}\left(F_{\infty}\right)^{\times[E: F]}
$$

taking $K_{\infty}$ to $K_{F \infty}^{\times[E: F]}$ and letting

$b_{E / F}(f)(g):=\int_{\mathrm{GL}_{2}\left(F_{\infty}\right)} \cdots \int_{\mathrm{GL}_{2}\left(F_{\infty}\right)} f\left(h_{1}, \ldots, h_{[E: F]-1}, h_{[E: F]-1}^{-1} h_{[E: F]-2}^{-1} \ldots h_{1}^{-1} g\right) d h_{1} \ldots d h_{[E: F]-1}$.

On the dense subspace

$$
\mathcal{C}\left(\mathrm{GL}_{2}\left(F_{\infty}\right) / / K_{F \infty}\right)^{\otimes[E: F]} \leq \mathcal{C}\left(G\left(F_{\infty}\right) / / K_{\infty}\right)
$$

this map just coincides with the [E:F]-fold convolution, and therefore (4.0.5) is a continuous algebra morphism. There is ambiguity in the choice of isomorphism (4.0.4), but since spherical Hecke algebras are commutative this does not affect the definition of the morphism $b_{E / F}$.

Lemma 4.1. Let $\pi$ be a cuspidal automorphic representation of $\mathrm{GL}_{2}\left(\mathbb{A}_{F}\right)$ with base change $\pi_{E}$ to $G\left(\mathbb{A}_{F}\right)$. Iff $\in \mathcal{C}\left(G\left(F_{\infty}\right) / / K_{\infty}\right)$, then $\operatorname{tr} \pi_{\infty}\left(b_{E / F}(f)\right)=\operatorname{tr} \pi_{E \infty}(f)$.

Proof. Under the isomorphism (4.0.4), the representation $\pi_{E \infty}$ corresponds to

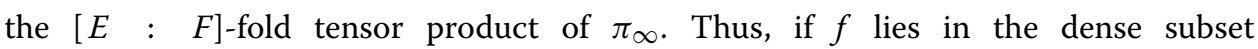
$C_{c}^{\infty}\left(\mathrm{GL}_{2}\left(F_{\infty}\right) / / K_{F \infty}\right)^{\otimes[E: F]}$, then the identity is trivial. The general case follows from the continuity of the distributions $\operatorname{tr} \pi_{E}$ on $\mathcal{C}\left(G\left(\mathbb{A}_{F}\right)^{1}\right)$ and $\operatorname{tr} \pi$ on $\mathcal{C}\left(\mathrm{GL}_{2}\left(\mathbb{A}_{F}\right)^{1}\right)$.

The considerations above yield continuous algebra morphisms

$$
\begin{aligned}
b_{E / F}: & \mathcal{C}\left(G\left(F_{\infty}\right) / / K_{\infty}\right) \otimes C_{c}^{\infty}\left(G\left(\mathbb{A}_{F}^{\infty}\right) / / K^{\infty}\right) \\
& \longrightarrow \mathcal{C}\left(G_{2}\left(F_{\infty}\right) / / K_{F \infty}\right) \otimes C_{c}^{\infty}\left(G_{2}\left(\mathbb{A}_{F}^{\infty}\right) / / G_{2}\left(\widehat{\mathcal{O}}_{F}\right)\right) .
\end{aligned}
$$

Lemma 4.2. The map (4.0.6) extends to a continuous algebra morphism

$$
b_{E / F}: \mathcal{C}\left(G\left(\mathbb{A}_{F}\right)^{1} / / K^{\infty}\right) \longrightarrow \mathcal{C}\left(G_{2}\left(\mathbb{A}_{F}\right)^{1} / / \mathrm{GL}_{2}\left(\widehat{\mathcal{O}}_{F}\right)\right)
$$

Moreover, if $\pi$ is a cuspidal automorphic representation of $\mathrm{GL}_{2}\left(\mathbb{A}_{F}\right)$ with cuspidal base change $\pi_{E}$ to $\mathrm{GL}_{2}\left(\mathbb{A}_{E}\right)$ and $f \in \mathcal{C}\left(G\left(\mathbb{A}_{F}\right)^{1} / / K^{\infty}\right)$ then

$$
\operatorname{tr} \pi_{E}(f)=\operatorname{tr} \pi\left(b_{E / F}(f)\right) .
$$


Proof. By Lemma 4.1, $b_{E / F}$ is a continuous algebra morphism on the dense subspace

$$
\mathcal{C}\left(G\left(F_{\infty}\right) / / K_{\infty}\right) \otimes C_{c}^{\infty}\left(G\left(\mathbb{A}_{F}^{\infty}\right) / / K^{\infty}\right) \cap \mathcal{C}\left(G\left(\mathbb{A}_{F}\right)^{1} / / K\right)
$$

of $\mathcal{C}\left(G\left(\mathbb{A}_{F}\right)^{1} / / K\right)$. Since $\mathcal{C}\left(G\left(\mathbb{A}_{F}\right) / / K\right)$ is a Frechet space, $b_{E / F}$ therefore extends uniquely to a continuous algebra morphism. To deduce the final assertion of the lemma, we invoke the continuity of the distributions $\operatorname{tr} \pi_{E}$ on $\mathcal{C}\left(G\left(\mathbb{A}_{F}\right)^{1}\right)$ and $\operatorname{tr} \pi$ on $\mathcal{C}\left(\mathrm{GL}_{2}\left(\mathbb{A}_{F}\right)^{1}\right)$ together with Lemma 4.1.

\section{The construction of $V$}

In this section, we construct $V \in \mathcal{C}\left(G\left(\mathbb{A}_{F}\right)^{1}\right)$ and use it to prove our main theorem, Theorem 1.2. We assume that $E / F$ is a Galois extension of number fields that is everywhere unramified and split at all infinite places. We also assume that

$$
\operatorname{Gal}(E / F)=\langle\iota, \tau\rangle
$$

where $|\iota|=2$ and $\tau$ has odd order. We let $F^{\prime}$ be the fixed field of $\iota$ and $F_{0}$ the fixed field of $\tau$.

\subsection{The subgroup $H$}

Let $U_{2}$ be the unitary group over $\mathcal{O}_{F^{\prime}}$ whose points in an $\mathcal{O}_{F^{\prime}}$-algebra $R$ are given by

$$
U_{2}(R):=\left\{g \in \mathrm{GL}_{2}\left(\mathcal{O}_{E} \otimes_{\mathcal{O}_{F^{\prime}}} R\right): g=\left({ }_{1}{ }^{1}\right)^{\iota} g^{-t}\left({ }_{1}{ }^{1}\right)\right\} .
$$

Moreover, let

$$
H:=\operatorname{Res}_{\mathcal{O}_{F^{\prime}} / \mathcal{O}_{F}} U_{2} \leq G .
$$

Recall that a measurable subset $\mathfrak{F} \leq H\left(\mathbb{A}_{F}\right)$ is a fundamental domain for the action of $H(F)$ if $H(F) \mathfrak{F}=H\left(\mathbb{A}_{F}\right)$ and $\gamma \mathfrak{F} \cap \mathfrak{F}=\emptyset$ for all $\gamma \in H(F)$.

Let $B_{H} \leq H$ be the Borel subgroup of upper-triangular matrices.

Lemma 5.1. There exists a fundamental domain $\mathfrak{F} \subseteq H\left(\mathbb{A}_{F}\right)$ contained in a set of the form

$$
\left\{\Omega\left({ }^{t}{ }^{-1}\right) K \cap H\left(\mathbb{A}_{F}\right): t>T\right\}
$$

for some $T>0$ and some compact subset $\Omega \leq B_{H}\left(\mathbb{A}_{F}\right)$.

Here, we regard the matrices $\left({ }^{t} t^{-1}\right), t \in \mathbb{R}^{\times}$as elements of $B_{H}\left(F_{\infty}\right)$ via the diagonal embedding.

Proof. This follows from [3] (Theorem 5.3) together with the Iwasawa decomposition.

Let $\mathfrak{F}$ be a fundamental domain as in Lemma 5.1 and let $\Psi \in C_{c}^{\infty}\left(G\left(\mathbb{A}_{F}\right)^{1} / / K\right)$. Moreover, for $g \in G\left(\mathbb{A}_{F}\right)$, let

$$
\mathbb{W}_{g}:=\prod_{w} \mathbb{W}_{g_{w}} .
$$

We define

$$
V:=V_{\Psi}:=\int_{\mathfrak{F}} \sum_{y \in E^{\times}} \Psi * \mathbb{W}\left(y_{1}{ }_{1}\right) d h .
$$


Of course, one must check that this sum is absolutely convergent, but this will be done in the course of the proof of Proposition 5.2 below.

Remark. The operator

$$
\sum_{y \in E^{\times}} \Psi * \mathbb{W}\left(y_{1}\right) h^{\prime}
$$

as a function of $h \in G\left(\mathbb{A}_{F}\right)$, is invariant under the subgroup of $G(F)$ of matrices of the form $\left(\begin{array}{cc}a & b \\ 1\end{array}\right)$ on the left and $K$ on the right. It is not invariant under all of $H(F)$ on the left, however, hence the need for a choice of fundamental domain. We have chosen our fundamental domain in order to guarantee the convergence of $V$. On the other hand, if one wanted to build a trace formula weighted by central $L$-values as mentioned in $\S 1.3$ one could probably do without choosing a specific fundamental domain.

Proposition 5.2. One has that $V \in \mathcal{C}\left(G\left(\mathbb{A}_{F}\right)^{1}\right)$.

We start with a lemma. For $m \in E_{\infty}$, let

$$
\|m\|_{\infty}:=\max _{w \mid \infty}\left(|m|_{w}\right) .
$$

Lemma 5.3. Let $1 \geq \alpha>0$ and let

$$
D:=\left\{m \in E_{\infty}:|m|_{\infty}>\alpha\right\} .
$$

Then for all $X, Y \in U(\mathfrak{g}), N \geq 0$, and $m \in D$, one has

$$
\left|X *\left(\Psi * \mathbb{W}\left(m_{1}\right)\right)_{\infty} * Y\right| \ll X, Y, \Psi, N, \alpha \min \left(1,\|m\|_{\infty}\right)^{-N}
$$

Proof. Let $m \in D$. If $|m|_{w} \geq 1$ for all $w \mid \infty$, then the lemma follows immediately from Lemma 3.4. If $|m|_{w} \leq 1$ for all $w \mid \infty$, then $|m|_{w}>\alpha$ for all $w \mid \infty$ and hence the lemma again follows from Lemma 3.4 .

Thus, we can assume without loss of generality that $m \in D$ satisfies $\|m\|_{\infty}>1$ and that there is a $w_{0} \mid \infty$ so that $|m|_{w_{0}}=\min _{w \mid \infty}\left(|m|_{w}\right)<1$. By assumption

$$
|m|_{w_{0}}>\frac{\alpha}{\prod_{\substack{w \mid \infty \\ w \neq w_{0}}}|m|_{w}} \geq \frac{\alpha}{\|m\|_{\infty}^{[E: \mathbb{Q}]-1}} .
$$

Applying Lemma 3.4, there is a $B>0$ such that for any $A>0$ one has

$$
\left|X *\left(\Psi * \mathbb{W}\left(\begin{array}{ll}
m_{1} &
\end{array}\right)\right)_{\infty} * Y\right| \ll_{A, X, Y, \Psi}\|m\|_{\infty}^{-A}\left(\frac{\alpha}{\|m\|_{\infty}^{[E: \mathbb{Q}]-1}}\right)^{-B([E: \mathbb{Q}]-1)}
$$

Taking $A \geq N+([E: \mathbb{Q}]-1)^{2} B$, we deduce the lemma.

Proof of Proposition 5.2. Let $X, Y \in U(\mathfrak{g})$. One has

$$
\begin{aligned}
& \|X * V * Y\|_{L^{1}\left(G\left(\mathbb{A}_{F}\right)^{1}\right)} \mid X * \int_{\mathfrak{F}} \sum_{y \in E^{\times}} \Psi * \mathbb{W}\left(y_{1}{ }_{1}\right) h \\
& =\int_{G\left(\mathbb{A}_{F}\right)^{1}} d h * Y(g) \mid d g \\
& \leq \int_{G\left(\mathbb{A}_{F}\right)^{1}} \int_{\mathfrak{F}} \sum_{y \in E^{\times}}\left|X * \Psi * \mathbb{W}\left(y_{1}{ }_{1}\right) * Y\right|(g) d h d g .
\end{aligned}
$$


Here, we are differentiating under the integral sign. This is justified if we show that the latter expression is convergent, which is what we will prove.

Using the Iwasawa decomposition, we write a general element of $H\left(\mathbb{A}_{F}\right)$ as

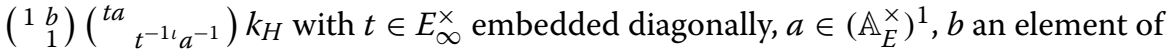

$$
\left\{x \in \mathbb{A}_{E}: \operatorname{tr}_{E / F^{\prime}}(x)=0\right\},
$$

and $k_{H} \in K \cap H\left(\mathbb{A}_{F}\right)$. With respect to this decomposition the Haar measure decomposes as

$$
d h=\frac{d b d t^{\times} d a^{\times} d k_{H}}{|t|_{\infty}}
$$

where $d b$ is a translation invariant measure on the 1-dimensional free $\mathbb{A}_{F}$-module (5.1.3) and $d k_{H}$ is a Haar measure on $K \cap H\left(\mathbb{A}_{F}\right)$. Thus, the integral above is equal to

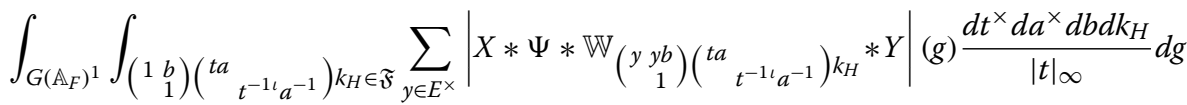

$$
\begin{aligned}
& \leq \int_{G\left(\mathbb{A}_{F}\right)} \int_{\left(\begin{array}{c}
1 \\
1
\end{array}\right)\left({ }^{t a}{ }_{t^{-1} a^{-1}}\right)} \sum_{k_{H} \in \mathfrak{F}} \sum_{y \in E^{\times}}\left|X * \Psi * \mathbb{W}_{\left(\begin{array}{c}
y y b \\
1
\end{array}\right)\left({ }^{t a}{ }^{-1 t^{-1} a^{-1}}\right) k_{H}} * Y\right|(g) \frac{d t^{\times} d a^{\times} d b d k_{H}}{|t|_{\infty}} d g .
\end{aligned}
$$

As above, let $U \leq \mathrm{GL}_{2}$ be the unipotent radical of the Borel subgroup of upper-triangular matrices. Replacing $h$ by $z u h k$ for $(z, u, k) \in Z_{G}\left(\mathbb{A}_{F}\right) \times \operatorname{Res}_{\mathcal{O}_{E} / \mathcal{O}_{F}} U\left(\mathbb{A}_{F}\right) \times K$ replaces $\mathbb{W}_{h}(g)$ by a scalar of norm 1 independent of $g$ times $\mathbb{W}_{h}\left(z^{-1} g\right)$ and hence

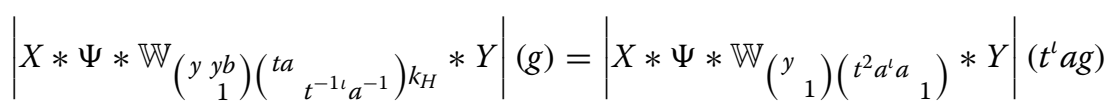

By our choice of $\mathfrak{F}_{H}$ (see Lemma 5.1), the integrals over $a, b$ and $k_{H}$ in (5.1.4) are over compact sets, and the integral over $t$ can be taken to be over $t>T$ for some fixed real number $T \in \mathbb{R}_{>0}$. Thus, (5.1.4) is bounded by a constant times

$$
\int_{G\left(\mathbb{A}_{F}\right)} \iint_{t=T}^{\infty} \sum_{y \in E^{\times}}\left|X * \Psi * \mathbb{W}\left(\begin{array}{l}
y \\
{ }_{1}
\end{array}\right)\left(t^{2} a^{l} a_{1}\right) * Y\right|(g) \frac{d t^{\times} d a^{\times}}{|t|_{\infty}} d g
$$

where the integral on $a$ is over a compact subset of $\left(\mathbb{A}_{E}^{\times}\right)^{1}$.

By definition,

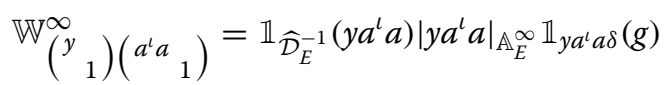

which vanishes identically if $\left(y a^{\imath} a\right)^{\infty} \notin \widehat{\mathcal{D}}_{E}^{-1}$. Here, $\delta \in \widehat{\mathcal{O}}_{E} \cap \mathbb{A}_{E}^{\times}$is a generator for $\mathcal{D}_{E}$. Thus, since the integral over $a$ in (5.1.5) is compactly supported, the sum over $y$ can be truncated to the subset $y \in \mathfrak{a}^{-1}-0$ for some ideal $\mathfrak{a} \subseteq \mathcal{O}_{E}$.

We consider the contribution of the nonarchimedian places to (5.1.5). For any $m \in$ $\left(\mathbb{A}_{E}^{\infty}\right)^{\times} \cap \widehat{\mathcal{O}}_{E}$, one has

$$
\left\|\Psi^{\infty} * \mathbb{1}_{m}\right\|_{L^{1}\left(G\left(\mathbb{A}_{F}^{\infty}\right)\right)} \leq\left\|\Psi^{\infty}\right\|_{L^{1}\left(G\left(\mathbb{A}_{F}^{\infty}\right)\right)}\left\|\mathbb{1}_{m}\right\|_{L^{1}\left(G\left(\mathbb{A}_{F}^{\infty}\right)\right)},
$$

so it suffices to bound $\left\|\mathbb{1}_{m}\right\|_{L^{1}\left(G\left(\mathbb{A}_{F}^{\infty}\right)\right)}$. Let $\varepsilon>0$. Recall that $\mathbb{1}_{m}$ can be written as the sum of the characteristic functions of $O_{\varepsilon}\left(|m|^{-1-\varepsilon}\right)$ disjoint right (or left) cosets of $K^{\infty}$ (compare [16]§ 4.2). Thus,

$$
\int_{G\left(\mathbb{A}_{F}^{\infty}\right)} \mathbb{1}_{m}(g) d g \ll_{\varepsilon}|m|_{\mathbb{A}_{E}^{\infty}}^{-1-\varepsilon}
$$


for $m \in \widehat{\mathcal{O}}_{E} \cap \mathbb{A}_{E}^{\times}$, and hence (5.1.5) is bounded by a constant depending on $\varepsilon$ times

$$
\iint_{t=T}^{\infty} \sum_{y \in \mathfrak{a}^{-1}-0} \int_{G\left(F_{\infty}\right)}\left|X *\left(\Psi * \mathbb{W}\left(y t^{2} a^{\imath} a_{1}\right)\right)_{\infty} * Y\right|(g)\left|y a^{\iota} a\right|_{\infty}^{\varepsilon} \frac{d g d t^{\times} d a^{\times}}{|t|_{\infty}}
$$

Since the integral over $a$ in (5.1.7) is over a compact set we can apply Lemma 5.3 to see that (5.1.7) is bounded by a constant depending on $N$ times

$$
\iint_{t=T}^{\infty} \sum_{y \in \mathfrak{a}^{-1}-0} \min \left(1,\left\|y t^{2} a^{\iota} a\right\|_{\infty}\right)^{-N}\left|y a^{\iota} a\right|_{\infty}^{\varepsilon} \frac{d t^{\times} d a^{\times}}{|t|_{\infty}}
$$

Provided that $N$ is sufficiently large this is bounded by a constant depending on $N$ times

$$
\int_{t=T}^{\infty} \sum_{y \in \mathfrak{a}^{-1}-0} \min \left(1,|| y t^{2} \|_{\infty}\right)^{-N}|y|_{\infty}^{\varepsilon} \frac{d t^{\times}}{|t|_{\infty}} \ll_{N} \sum_{y \in \mathfrak{a}^{-1}} \min \left(1,\|y\|_{\infty}\right)^{-N}|y|^{\varepsilon} \ll_{N} 1 .
$$

Finally, we prove a proposition which motivated our definition of $V$ in the first place. Recall that if $\Pi$ is a cuspidal automorphic representation of $G\left(\mathbb{A}_{F}\right)^{1}$ distinguished by $H\left(\mathbb{A}_{F}\right)^{1}=H\left(\mathbb{A}_{F}\right)$ then it is the base change of a cuspidal automorphic representation $\Pi^{\prime}$ of $\mathrm{GL}_{2}\left(\mathbb{A}_{F^{\prime}}\right)^{1}$. Moreover, in this case, there is a certain $H\left(\mathbb{A}_{F}\right)$-invariant linear functional on the space of Whittaker functions of $\Pi_{S}$ with respect to $\psi_{S}$ denoted in [6] ( $\$ 3.3$ and Definition 10.1) by

$$
\alpha^{\Pi_{S}^{\prime}}:=\prod_{v^{\prime} \mid S} \alpha_{\left(\begin{array}{l}
v^{\prime} \\
{ }^{\prime}
\end{array}{ }^{\prime}\right)}^{{ }^{\prime}}
$$

where the product is over places $v^{\prime}$ of $F^{\prime}$ dividing $S$. Since $H$ is quasi-split, these linear functionals $\alpha^{\Pi_{S}^{\prime}}$ are nonzero by [6] (Corollary 10.3 and Remark 10.4). We note that these distributions depend on the choice of measure on $H(F) \backslash H\left(\mathbb{A}_{F}\right)$ (see loc. cit.).

Proposition 5.4. If $\Pi$ is a cuspidal automorphic representation of $G\left(\mathbb{A}_{F}\right)^{1}$, spherical at all places and $f \in C_{c}^{\infty}\left(G\left(\mathbb{A}_{F}\right)^{1} / / K^{S}\right)$ then

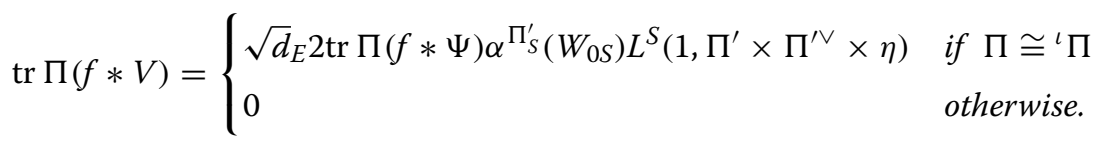

Here, $\Pi^{\prime}$ is a cuspidal automorphic representation of $G^{\prime}\left(\mathbb{A}_{F}\right)^{1}=G_{2}\left(\mathbb{A}_{F^{\prime}}\right)^{1}$ whose base change to $G\left(\mathbb{A}_{F}\right)^{1}$ is $\Pi, W_{O S} \in \mathcal{W}\left(\Pi_{S}, \psi_{S}\right)$ is the Whittaker newform, and $\eta: F^{\prime \times} \backslash \mathbb{A}_{F^{\prime}}^{\times} \longrightarrow$ $\mathbb{C}^{\times}$is the Kronecker character of $E / F^{\prime}$.

Proof. By construction, the operator $\Pi(V)$ projects the space of $\Pi$ onto the line spanned by the Whittaker newform $\varphi$ of $\Pi$ as does $\Pi(\Psi)$. Thus,

$$
\begin{aligned}
\operatorname{tr} \Pi(f * V) & =\int_{G(F) \backslash G\left(\mathbb{A}_{F}\right)^{1}} \frac{\left(\Pi\left(f * \int_{\mathfrak{F}} \sum_{y \in E^{\times}} \Psi * \mathbb{W}\left(y_{1}{ }_{1}\right) h\right) \varphi\right)(g) \bar{\varphi}(g)}{\|\varphi\|^{2}} d g \\
& =\int_{\mathfrak{F}} \sum_{y \in E^{\times}} W^{\varphi}\left(\left(\begin{array}{ll}
y_{1} \\
1
\end{array}\right) h\right) d h \int_{G(F) \backslash G\left(\mathbb{A}_{F}\right)^{1}} \frac{(\Pi(f * \Psi) \varphi)(g) \bar{\varphi}(g)}{\|\varphi\|^{2}} d g \\
& =\operatorname{tr} \Pi(f * \Psi) \int_{\mathfrak{F}} \sum_{y \in E^{\times}} W^{\varphi}\left(\left(\begin{array}{ll}
y_{1} \\
1
\end{array}\right) h\right) d h .
\end{aligned}
$$


Recognizing the Whittaker expansion in the expression above (compare (3.1.4)) we obtain

$$
\int_{\mathfrak{F}} \sum_{y \in E^{\times}} W^{\varphi}\left(\left({ }^{y}{ }_{1}\right) h\right) d h=\sqrt{d}_{E} \int_{H(F) \backslash H\left(\mathbb{A}_{F}\right)} \varphi(h) d h .
$$

The proposition now follows from [6] (Theorem 10.2).

\subsection{Proof of Theorem 1.2}

We assume the notation of $\S 1.2$. We have that

$$
\begin{aligned}
\operatorname{tr} R_{\text {cusp }}\left(f^{G_{0}} * b_{E / F_{0}}(V)\right) & =\sum_{\pi_{0}} \operatorname{tr} \pi_{0}\left(f^{G_{0}} * b_{E / F_{0}}(V)\right) \\
& =\sum_{\pi_{0}} \operatorname{tr} \pi_{0}\left(f^{G_{0}}\right) \operatorname{tr} \pi_{0}\left(b_{E / F_{0}}(V)\right)
\end{aligned}
$$

where the (absolutely convergent) sum is over all cuspidal automorphic representations of $G_{0}\left(\mathbb{A}_{F}\right)^{1}$. Recall that each $\pi_{0}$ admits a unique cuspidal base change $\pi_{0 E}$ to $G\left(\mathbb{A}_{F}\right)$, and $\pi_{0 E} \cong \sigma_{0 E}$ if and only if $\pi_{0} \cong \sigma_{0} \otimes \chi$ for some character $\chi$ of $F_{0} \backslash \mathbb{A}_{F_{0}}^{\times}$trivial on $\mathrm{N}_{E / F_{0}}\left(\mathbb{A}_{F_{0}}^{\times}\right)$. This was proven by Langlands [17] in the prime degree case, and for the deduction of the general case, which is easy since the extension $E / F^{\prime}$ has odd degree, see [19].

Thus, by Lemma 4.2, the sum (5.2.1) is equal to

$$
\sum_{\pi_{0}} \operatorname{tr} \pi_{0 E}(f) \operatorname{tr} \pi_{0 E}(V)=\left[E: F_{0}\right] \sum_{\Pi: \Pi \cong \Pi^{\tau}} \operatorname{tr} \Pi(f) \operatorname{tr} \Pi(V)
$$

where the latter sum is over all cuspidal automorphic representations of $G\left(\mathbb{A}_{F}\right)^{1}$ invariant under $\tau$. The theorem now follows from Proposition 5.4.

\section{Acknowledgements}

The authors thank R. Langlands, B. C. Ngô, and P. Sarnak for their generous help and encouragement over the past few years, and E. Lapid for pointing out a mistake in an earlier version of this paper that lead to the simpler approach exposed here. The first author also thanks $\mathrm{H}$. Hahn for her constant encouragement and help with proofreading. Finally, the authors thank the referees for useful comments and corrections which improved the exposition.

The first author is thankful for partial support provided by NSF grant DMS-1405708. Any opinions, findings, and conclusions or recommendations expressed in this material are those of the author and do not necessarily reflect the views of the National Science Foundation.

\section{Author details}

${ }^{1}$ Department of Mathematics, Duke University, Durham, NC 27708-0320, USA. ² Department of Mathematics, University of Chicago, Chicago, IL 60637, USA.

Received: 18 November 2014 Accepted: 31 March 2015

Published online: 03 August 2015

\section{References}

1. Arthur, J: The Endoscopic Classification of Representations: Orthogonal and Symplectic Groups. AMS, Providence (2013)

2. Arthur, J, Clozel, L: Simple Algebras, base change, and the advanced theory of the trace formula. Princeton University Press (1989)

3. Borel, A: Automorphic forms on reductive groups. In: Automorphic Forms and Applications. AMS, Providence, (2007)

4. Cogdell, J: $L$-functions and converse theorems for $\mathrm{GL}_{n}$. In: Automorphic Forms and Applications. AMS, (2007)

5. Cogdell, JW, Kim, HH, Piatetski-Shapiro, II, Shahidi, F: Functoriality for the classical groups. Publ. Math. Inst. Hautes Études Sci. 99, 163-233 (2004)

6. Feigon, B, Lapid, E, Offen, O: On representations distinguished by unitary groups. Publ. Math. Inst. Hautes Études Sci. $\mathbf{1 1 5}(1), 185-323$ (2012)

7. Finis, T, Lapid, E: On the Arthur-Selberg trace formula for GL(2). Groups Geometry Dynamics. 5, 367-391 (2011)

8. Finis, T, Lapid, E: On the continuity of Arthur's trace formula: the semisimple terms. Compositio Math. 147, 784-802 (2011)

9. Finis, T, Lapid, E, Müller, W: On the spectral side of Arthur's trace formula-absolute convergence. Ann. Math. 174 173-195 (2011)

10. Gelbart, S: Automorphic Forms on Adele Groups. Princeton University Press, Princeton (1975) 
11. Getz, JR: An approach to nonsolvable base change and descent. J. Ramanujan Math. Soc. 27, 143-211 (2012)

12. Godement, R, Jacquet, H: Zeta Functions of Simple Algebras. Springer (1972)

13. Guralnick, R, Malle, G: Products of conjugacy classes and fixed point spaces. J. Am. Math. Soc. 25, 77-121 (2012)

14. Herman, PE: Quadratic base change and the analytic continuation of the Asai $L$-function: a new trace formula approach, preprint. arXiv:1008.3921v4

15. Jacquet, H, Langlands, R: Automorphic forms on GL(2). Springer (1970)

16. Knightly, A, Li, C: Petersson's trace formula and the Hecke eigenvalues of Hilbert modular forms. In: Modular Forms on Schiermonnikoog. Cambridge Univ. Press 92008

17. Langlands, RP: Base Change for GL(2). Princeton (1980)

18. Langlands, RP: Beyond endoscopy. In: Contributions to Automorphic Forms, Geometry, and Number Theory: A Volume in Honor of Joseph Shalika. The Johns Hopkins University Press, (2004)

19. Lapid, E, Rogawski, J: On twists of cuspidal representations of GL(2). Forum Math. 10, 175-197 (1998)

20. Laumon, G: Cohomology of Drinfeld Modular Varieties, Part I: Geometry, Counting of Points and Local Harmonic Analysis. Cambridge (1996)

21. Lafforge, L: Noyaux du transfert automorphe de Langlands et formules de Poisson non linéaires. Japan J. Math. 9(1), $1-68$ (2014)

22. Mok, CP: Endoscopic classification of representation of quasi-split unitary groups. Memoirs of the American Mathematical Society. 235(1108) (2015)

23. Popa, A: Whittaker newforms for archimedian representations of GL(2). J. Number Theory 128, 1637-1645 (2008)

24. Sarnak, P: Comments on Robert Langlands' lecture: "Endoscopy and Beyond", Available at. publications.ias.edu/ sarnak

25. Schmidt, R: Some remarks on local newforms for GL(2). J. Ramanujan Math. Soc. 17, 115-147 (2002)

26. Venkatesh, A: Beyond endoscopy and the classification of special forms on $\mathrm{GL}(2)$. J. für die reine und angew. Math $\mathbf{5 7 7}, 23-80(2004)$

27. White, PJ: The base change $L$-function for modular forms and beyond endoscopy. J. Number Theory. 140, 1337 (2014)

28. Zhang, SW: Gross-Zagier formula for GL(2). Asian J. Math. 5, 183-290 (2001)

\section{Submit your manuscript to a SpringerOpen ${ }^{\circ}$ journal and benefit from:}

- Convenient online submission

- Rigorous peer review

- Immediate publication on acceptance

- Open access: articles freely available online

- High visibility within the field

Retaining the copyright to your article

Submit your next manuscript at $\gg$ springeropen.com 